\title{
DIAGNOSIS OF HEART DISEASE USING FEATURE SELECTION METHODS BASED ON RECURRENT FUZZY NEURAL NETWORKS
}

\author{
Shirin Kordnoori*1 | Hamidreza Mostafaei ${ }^{2}$ | Mohsen Rostamy-Malkhalifeh ${ }^{3}$ | Mohammadmohsen \\ Ostadrahimi $^{3}$ | Saeed Agha Banihashem ${ }^{4}$
}

\footnotetext{
${ }^{1}$ Dept. of Computer Engineering-Artificial Intelligenc, Islamic Azad University, Tehran, Iran

${ }^{2}$ Dept. of Statistics, Islamic Azad University, Tehran, Iran

${ }^{3}$ Dept. of Mathematics, Islamic Azad University, Tehran, Iran

${ }^{4}$ School of International Relations, Ministry of Foreign Affairs of the Islamic Republic of Iran, Tehran, Iran
}

\section{Correspondence}

*Shirin Kordnoori, Department of Computer Engineering-Artificial Intelligence, North Tehran Branch, Islamic Azad University, Tehran, Iran Email:

sh.kordnoori@iau-tnb.ac.ir

\section{Present Address}

Department of Computer EngineeringArtificial Intelligence, North Tehran Branch, Islamic Azad University, Vafadar Blvd., Shahid Sadoughi St., Hakimiyeh Exit, Shahid Babaee Highway, Tehran, Iran

\begin{abstract}
The World Health Organization (WHO) estimated one-third of all global deaths reason by cardiovascular diseases. Nowadays, artificial intelligence attracts many considerations in diagnosing heart disease. This study used trained recurrent fuzzy neural networks (RFNN) for diagnosing heart disease. This study also used five kinds of feature selection and extraction models for comparing the action of a model, such as data envelopment analysis (DEA), Linear Discriminative Analysis (LDA), Principle Component Analysis (PCA), Correlation Feature Selection (CFS), and Relief. By using these methods, this paper diagnosed whether the patient has a heart disease problem or not. The results showed that Correlation feature selection has the best operation in feature selection in RFNN by accuracy of $98.4 \%$.
\end{abstract}

\section{KEYWORDS:}

Artificial Intelligent, Feature Selection Model, Heart Disease, Recurrent Fuzzy Neural Networks

\section{1 | INTRODUCTION}

When the heart muscle doesn't pump blood as well as it should, heart failure, sometimes known as congestive heart failure, occurs. Certain conditions, such as narrowed arteries in your heart or high blood pressure, gradually leave your heart too weak or stiff to fill and pump efficiently. Congestive heart failure represents $31 \%$ of the overall deaths. Around 17.7 million people 
lost their lives from cardiovascular diseases in 2015, which the World Health Organization (WHO, 2017). It is estimated that 7.4 million deaths are caused by coronary heart disease (13\% of overall deaths), and 6.7 million are due to stroke.

Advances in the medical field help anticipate the possibility of heart failures that patients can come across later in their lives. It is strictly advised that all people, including the ones feeling perfectly well, should see a heart specialist twice a year to investigate the possible existence of any evidence that may cause heart failure. Nowadays, artificial intelligence attracts many considerations in diagnosing heart disease because the intelligence method is faster and has fewer mistakes in its detection than traditional human methods. These techniques used for diagnosing diseases are artificial neural network (ANN), fuzzy logic, neuro-fuzzy methods, machine learning, etc. Detecting lung cancer or strokes, assessing the risk of sudden cardiac death or other heart diseases, Classifying skin lesions in skin images, and Finding indicators of diabetic retinopathy in eye images are the latest achievements of $\mathrm{AI}$ in medicine.

In this study, based trained recurrent fuzzy neural networks (RFNN) has used for diagnosing heart disease and for comparing the action of this method five kinds of feature selection/extraction models such as data envelopment analysis (DEA), Linear Discriminative Analysis (LDA), Principle Component Analysis (PCA), Correlation Feature Selection (CFS) and Relief has used. This study aims to find the right feature selection method for RFNN best accuracy. The problem of the previous works was that they were confined with simple neural networks or limited feature selection algorithms and reached low accuracies. This study tries to resolve these limitations and reach the best accuracy by finding the right feature selection method.

\section{1 | DEA Feature Selection}

One of the well-known methods to calculate the efficiency of Decision-making units (DMUs) is data envelopment analysis (DEA). This method is non-parametric based on linear programming was first proposed by Rakhshan ${ }^{[1]}$, Barat et al. ${ }^{[2]}$. There are two different methods to solve this problem; one can be output-oriented or input-oriented. In this paper, output-oriented has been used for the feature selection method, which is discussed in the proposed method.

Using the DEA model as a feature selection method, it is important to consider each attribute as a Decision-Making Unit (DMUs). The assumption is that each DMU is transformed by consuming resources as inputs try to produce some outputs. According to Azadeh et al., "DEA models can be input or output-oriented and can be specified as constant returns to scale (CRS) or variable returns to scale (VRS) or BCC model. Bellow the basic DEA model known as the BCC model is presented for the se $\neg$ lec $\neg$ ted enᄀtity k:

$$
\begin{gathered}
E_{P}=\max \frac{\sum_{i}^{s} u_{i} y_{i k}}{\sum_{j}^{m} v_{j} x_{j k}+v_{0}} \\
\text { s.t }: \frac{\sum_{i}^{s} u_{i} y_{i l}}{\sum_{j}^{m} v_{j} x_{j l}+v_{0}}, \leq 1 \quad \forall l, u_{i} \geq 0, \quad v_{j} \geq 0, \quad v_{0}=\text { free }
\end{gathered}
$$

where:

s: the number of outputs

$u_{i}$ : the weights to be determined for output $i$

$y_{i k}$ : the observed magnitude of $i$-type output for entity $k v_{j}$ : the weights to be determined for input $j$

$x_{j k}$ : the observed magnitude of $j$-type input for entity $k$

$m$ : the number of inputs

There are two different methods for BCC models, one being output-oriented and the Input-oriented BCC model. Here, the first method by placing a numerator equal to 1 is chosen. In the following, an output-oriented BCC model presents:

$$
\begin{gathered}
E_{P}=\min \Sigma_{j}^{m} v_{j} x_{j k}+v_{0} \\
\text { s.t }: \Sigma_{i}^{s} u_{i} y_{i k}=1
\end{gathered}
$$




$$
\Sigma_{i}^{s} u_{i} y_{i l} \leq \Sigma_{j}^{m} v_{j} x_{j l}+v_{0}, \quad \forall l, u_{i} \geq 0, \quad v_{j} \geq 0, \quad v_{0}=\text { free }
$$

In this study, the output of DMUs is equal to entropy value gain, which computes with Shannon's entropy. At first, the decision matrix normalizes:

$$
P_{i j}=\frac{x_{i j}}{\sum_{j=1}^{m} x_{i j}}, j=1,2, \ldots, m \quad i=1,2, \ldots, n
$$

The entropy calculates by using the following formula:

$$
E_{j}=-k \sum_{i=1}^{m}\left[P_{i j} \ln _{i j} P_{i j}\right], \quad \forall j=1,2, \ldots, n, \quad k=\frac{1}{\ln (m)}
$$

The degree of deviation of each criterion from its entropy's value calculates:

$$
d_{j}=1-E_{j}
$$

Finally, the degree of deviation of each criterion from its entropy's value calculate:

$$
w_{j}=\frac{d_{j}}{\sum_{j=1}^{n} d_{j}}, \quad \forall j=1,2, \ldots, n
$$

Applying Data Envelopment Analysis and Shannon's entropy method for selecting the features make this model compared with the other method. The Advantage of the proposed system is that the feature extraction and selection techniques are used to optimize fuzzy recurrent neural networks.

In this paper, first, the methods about how to use feature selection proposed system and second about dataset which has used in this work are presented in continue simulation results. And finally, the conclusion and suggestion for future works are described.

\section{2 | PREVIOUS RESEARCHES}

Samuel et al. ${ }^{[3]}$ in 2017 presented a decision support system based on ANN and Fuzzy-AHP to predict possible risks of heart failure. They compared their results with the conventional ANN and seven previous works to denote that their approach had better accuracy.

Uyar and Ilhan ${ }^{[4]}$ in 2017, used a GA-based trained RFNN approach on the UCI Cleveland dataset to diagnose heart disease. The result of the approach was evaluated with RMSEs, sensitivities, specificities, precisions, F-scores, PMEs, and accuracies of the training set, testing set, and overall. This study achieved a testing set (45 instances) accuracy of $97.78 \%$ and overall accuracy of $96.63 \%$. The RFNN used in this study has 13 inputs, seven hidden neurons, and one output neuron. The weights and biases of the RFNN were coded as 64 bits long genes. GA used with mutation probability 0.05 , multi-point crossover with probability 0.25 , and size of population 100 .

Louridi et al. ${ }^{[5]}$ in 2019 , compared machine learning algorithms with different performance metrics and improved their accuracy. Their approach consists of using the mean value to replace missing in the preprocessing step. The results proved that replacing inexistent values with the mean performs well. The score accuracy of $86,8 \%$ was obtained by using SVM with a linear kernel. Louridi et al. ${ }^{[5]}$ Buettner and Schunter in 2019 presented an approach to heart disease classification, using the Random Forest machine learning algorithm and attributes based on clinical data and patient test results. It reached an overall accuracy of $84.448 \%$.

Yadav et al. [6] in 2020, predicted heart disease and then analyzed the treatment for the disease by different algorithms, such as the Naive Bayes, the K-nearest neighbors, and logistic regression. Alongside simple algorithms, the hybrid algorithms were generated in their research paper. 


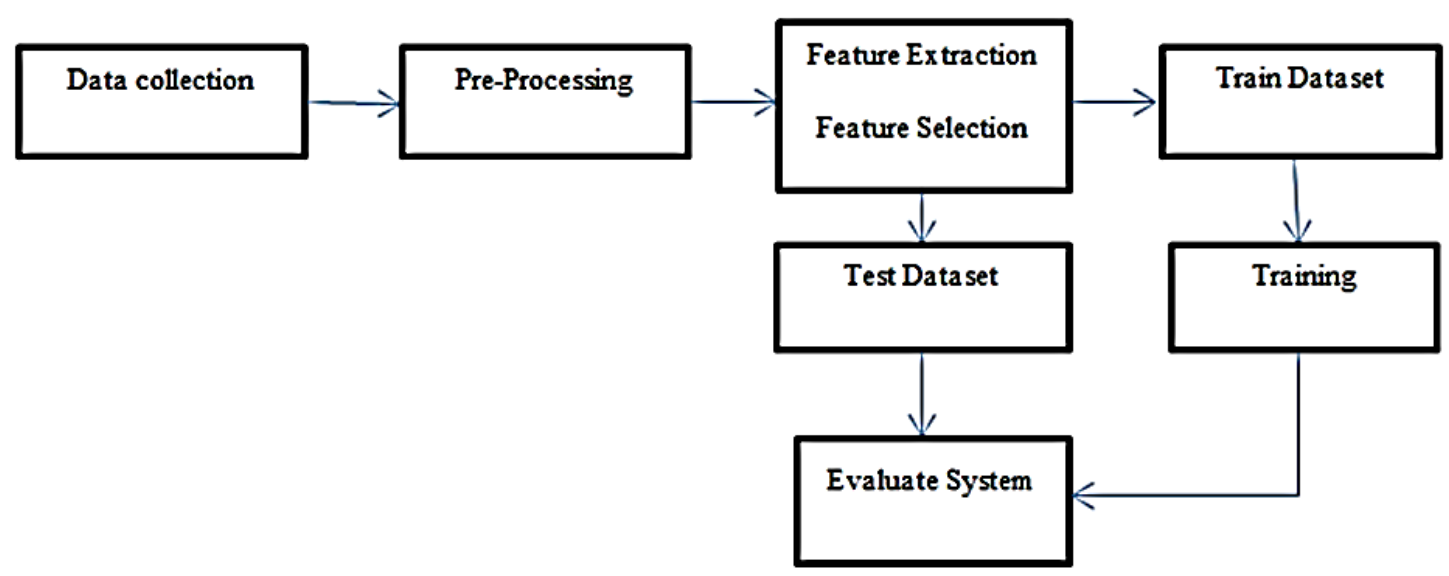

FIGURE 1 The diagram of the proposed diagnosing heart disease method.

Reddy et al. ${ }^{[7]}$ in 2020 , calculated the accuracy of machine learning algorithms for predicting heart disease. The algorithms are k-nearest neighbor, decision tree, linear regression, and support vector machine(SVM). The study used the UCI repository dataset for training and testing. accuracy, value has been calculated, and it concluded that KNN was the best among them with $87 \%$ accuracy.

Lin et al. ${ }^{[8]}$ in 2020, used both NN and CNN machine learning models to solve the heart disease diagnosis problems. Using the Cleveland Heart Disease Data Set, they compared the performance of the two machine learning models by adjusting the parameter settings and conducting a series of experiments.

Sharma et al. [9] in 2020, in their research, explored neural networks, where it had analyzed optimizing algorithms and weight initializing techniques. They used common algorithms and observed their effect on the accuracy of the model. The coronary heart disease dataset was used in their work.

Li et al. [10] in 2020 proposed a system to diagnosis heart disease based on machine learning techniques and classification algorithms. The study included support vector machine, logistic regression, convolution neural network, K-nearest neighbor, Naïve bays, and Decision tree. While standard features selection algorithms have been used, such as Relief, Minimal redundancy maximal relevance, least absolute shrinkage selection operator, and Local learning for removing irrelevant and redundant features. Finally, the proposed feature selection algorithm to solve the feature selection problem.

Magesh and Swarnalatha ${ }^{[11]}$ in 2020, presented a cluster-based DT learning (CDTL) approach for heart disease classification using the large distribution samples and an entropy-based partition. Their class-based partition clustering approach reduced $61.53 \%$ of feature dimensionality without sacrificing prediction accuracy.

\section{3 | MATERIAL AND METHOD}

As mentioned in the previous section, the seven steps should be followed to diagnose heart disease using recurrent fuzzy neural networks; the proposed method will be explained in the rest of this section. The diagram of these steps is depicted in Figure 1

Step1: Data collection: This step involves collecting data. As mentioned, the Cleveland dataset is used to simulate the proposed model. This dataset will be introduced in the rest of the paper. Step2: Pre-processing data: At this step, the diagnosis of heart disease attribute is categorized into two classes, denoted as absence and presence of the heart disease. There are six missing values in the dataset. Therefore, this data must be normalized and assigned a label after normalization.

Step3: Feature extraction/feature selection: The dataset contains many raw continuous and discrete features. The system is more efficient by using feature extraction methods, the quality of the extracted feature, and reducing the new data's dimensions. Feature extraction is important because the selected features determine the accuracy of the system. The features considered in 
TABLE 1 Attributes of the UCI Cleveland dataset.

\begin{tabular}{cll}
\hline No & Name & Definition \\
\hline 1 & age & Age in years \\
2 & sex & Sex \\
3 & cp & Chest pain type \\
4 & trestbps & Resting blood pressure on admission to the hospital in $\mathrm{mm} \mathrm{Hg}$ \\
5 & chol & Serum cholesterol in $\mathrm{mg} / \mathrm{dl}$ \\
6 & fbs & Fasting blood sugar is greater than $120 \mathrm{mg} / \mathrm{dl}$ or not \\
7 & restecg & Resting electrocardiographic results \\
8 & thalach & Maximum heart rate achieved \\
9 & exang & Exercise-induced angina \\
10 & oldpeak & ST depression induced by exercise relative to rest \\
11 & slope & The slope of the peak exercise ST segment \\
12 & ca & Number of major vessels $(0-3)$ colored by fluoroscopy \\
13 & thal & The heart status \\
14 & num & Diagnosis of heart disease $(0=$ healthy; $1=$ Sick1;2 $=$ \\
& & Sick2; 3= Sick3; 4=Sick4) \\
\hline
\end{tabular}

this section include the mean, minimum, maximum, deviation of the criterion, variance, and skewness. Selecting an appropriate set of features to represent the main information of the original datasets is an important factor that influences the accuracy of classification methods. Enhancing the classification accuracy and predictability ability, increasing the training process speed, and decreasing the storage demands are some of the potential advantages of feature selection algorithms. The selected features in the previous step are divided into two parts and entered as inputs into the recurrent fuzzy neural network. These parts are Train Dataset and Test Dataset.

Step4: Train Dataset: The model initially fits on a training dataset, a set of examples used to fit the model's parameters (e.g., weights of connections between neurons in artificial neural networks). The model is trained on the training dataset using a supervised learning method.

Step5: Training: The model runs with the training dataset and produces results, then compared with the target for each input vector in the training dataset. Based on the comparison result and the specific learning algorithm being used, the model's parameters are adjusted.

Step6: Test Dataset: A test dataset is used to provide an unbiased evaluation of a final model fit on the training dataset. The data in the test dataset has never been used in the training phase.

Step7: Evaluate System: to analyze the performance of outputs according to the feature selections algorithm, the outputs compared with the targets. It should be noted that the evaluation measures for these classes are the accuracy, sensitivity, and specificity of the F-Score. To evaluate the results, a 5-fold cross-validation method has been used. During this process, the data are divided into five batches, and in each run, one batch is used as test data and four batches as training data.

Figure1. The diagram of the proposed diagnosing heart disease method

\section{1 | Dataset}

In this study, the Cleveland dataset (UCI, 1990) was used, which was received from the University of California Irvine (UCI) Machine Learning Repository heart disease dataset ${ }^{[12]}$. The Cleveland dataset has 303 instances of patient data, but 6 of them contain missing values. Table 1 shows the Cleveland dataset attributes with their definitions.

This dataset includes four independent databases contributed by four independent medical institutions that contain 76 attributes. In this paper, a subset of 14 of them has been used because:

- Age: Age is the most important risk factor in developing cardiovascular or heart diseases, with approximately a tripling of risk with each decade of life. Coronary fatty streaks can begin to form in adolescence. It is estimated that 82 percent of people who die of coronary heart disease are 65 and older. Simultaneously, the risk of stroke doubles every decade after age 55. 
- Sex: Men are at greater risk of heart disease than pre-menopausal women. Once past menopause, it has been argued that a woman's risk is similar to a man's, although more recent data from the WHO and UN disputes this. If a female has diabetes, she is more likely to develop heart disease than a male with diabetes.

- Angina (Chest Pain): Angina is chest pain or discomfort caused when your heart muscle doesn't get enough oxygen-rich blood. It may feel like pressure or squeezing in your chest. The discomfort also can occur in your shoulders, arms, neck, jaw, or back. Angina pain may even feel like indigestion.

- Resting Blood Pressure: Over time, high blood pressure can damage arteries that feed your heart. High blood pressure that occurs with other conditions, such as obesity, high cholesterol, or diabetes, increases your risk even more.

- Serum Cholesterol: A high level of low-density lipoprotein (LDL) cholesterol (the "bad" cholesterol) is most likely to narrow arteries. A high level of triglycerides, a type of blood fat related to your diet, also ups your risk of a heart attack. However, a high level of high-density lipoprotein (HDL) cholesterol (the "good" cholesterol) lowers your risk of a heart attack.

- Fasting Blood Sugar: Not producing enough of a hormone secreted by your pancreas (insulin) or not responding to insulin properly causes your body's blood sugar levels to rise, increasing your risk of a heart attack.

- Resting ECG: For people at low risk of cardiovascular disease, the USPSTF concludes with moderate certainty that the potential harms of screening with resting or exercise ECG equal or exceed the potential benefits. For people at intermediate to high risk, current evidence is insufficient to assess the balance of benefits and harms of screening.

- Max heart rate achieved: The increase in cardiovascular risk, associated with the heart rate acceleration, was comparable to the increase in risk observed with high blood pressure. It has been shown that an increase in heart rate by ten beats per minute was associated with an increase in the risk of cardiac death by at least $20 \%$, and this increase in the risk is similar to the one observed with an increase in systolic blood pressure by $10 \mathrm{~mm} \mathrm{Hg}$.

- Exercise-induced angina: The pain or discomfort associated with angina usually feels tight, gripping or squeezing, and can vary from mild to severe. Angina is usually felt in the center of your chest but may spread to either or both of your shoulders or your back, neck, jaw, or arm. It can even be felt in your hands. o Types of Angina a. Stable Angina / Angina Pectoris b. Unstable Angina c. Variant (Prinzmetal) Angina d. Microvascular Angina.

- Peak exercise ST segment: A treadmill ECG stress test is considered abnormal when horizontal or down-sloping ST-segment depression $\geq 1 \mathrm{~mm}$ at $60-80 \mathrm{~ms}$ after the J point. Exercise ECGs with up-sloping ST-segment depressions are typically reported as an 'equivocal' test. In general, the occurrence of horizontal or down-sloping ST-segment depression at a lower workload (calculated in METs) or heart rate indicates a worse prognosis and a higher likelihood of multi-vessel disease. The duration of ST-segment depression is also important, as prolonged recovery after peak stress is consistent with a positive treadmill ECG stress test. Another finding highly indicative of significant CAD is the occurrence of ST-segment elevation > $1 \mathrm{~mm}$ (often suggesting transmural ischemia); these patients are frequently referred urgently for coronary angiography. The number of major vessels, the heart status, and diagnosis of heart disease used in accruing heart dieses or not.

\section{2 | Evaluation Criteria}

The proposed approach performance is evaluated with sensitivity (Recall), specificity, F-score, and accuracy of the training set, testing set, and overall performance was analyzed by using the following equations. Respectively where Yi is actual, and Ri is the result of the ith diagnosis of heart disease attribute (num) obtained, True Negative (TN) is the prediction for the patients without heart disease that was found to have no heart disease, False Negative (FN) is the prediction for the patients without heart disease that was found to have heart disease, the True Positive (TP) is the prediction for the patients with a heart disease that were found to have heart disease. The False Positive (FP) is the prediction for the patients with heart disease with no heart disease.

$$
\begin{aligned}
& \text { Sensitivity }=\frac{T P}{T P+F N} \\
& \text { Specificity }=\frac{T N}{T N+F P}
\end{aligned}
$$




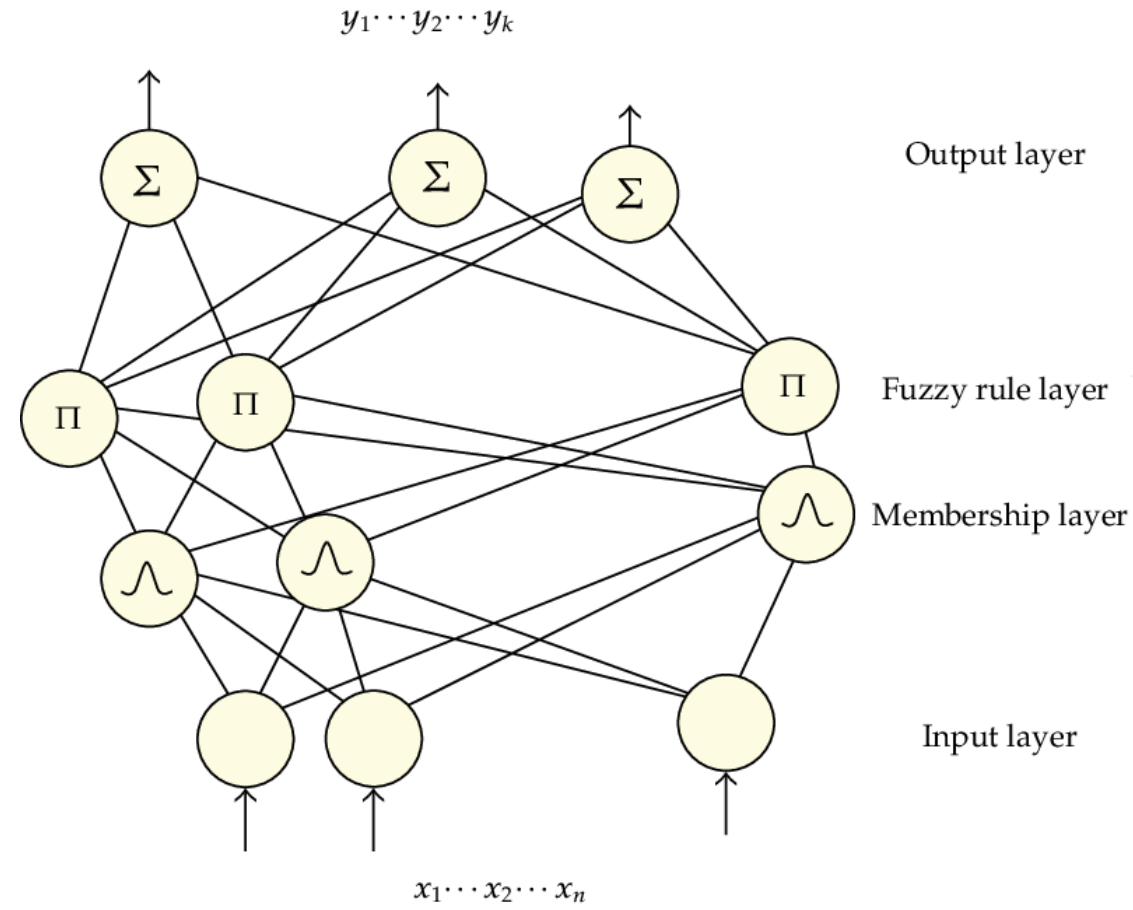

FIGURE 2 The structure of RFNN.

$$
\begin{gathered}
\text { Accuracy }=\frac{T P+T N}{T P+T N+F P+F N} \\
F \text {-score }=\frac{2 * \text { Recall } * \text { Precision }}{\text { Recall }+ \text { Precision }}
\end{gathered}
$$

Where:

$$
\text { Precision }=\frac{T P}{T P+F P}
$$

Also, the function of the classification algorithm depends directly on the size of the extracted features. The number of features must be minimized to develop the model's performance, and the dimensional effects must be reduced. One of the ways to reduce the size of the dimensions is feature selection algorithms. In this model, the feature selection is functional in determining which features are useful and differentiating between fall and non-fall activities. At this step, new features are introduced as inputs to classification algorithms. Five algorithms are used to select features: Data envelopment analysis linear discriminative analysis, Correlation attributes feature selection, Principal Component Analysis, and Relief.

\section{4 | RESULTS AND DISCUSSION}

The items were simulated in the MATLAB environment. The RFNN used in this study has 13 inputs, seven hidden neurons, and one output neuron, as shown in Figure 2 .

Feature extraction has applied to the dataset, and the extracted items were assigned to the feature selection algorithms to select the features. Table 2 shows these algorithms and their selected feature numbers gained by WEKA software. These features are introduced as inputs to the RFNN model. RFNN determine which input data belong to which heart disease or without heart disease classes: 
TABLE 2 Attributes of the UCI Cleveland dataset.

\begin{tabular}{ll}
\hline Method & Feature Numbers \\
\hline DEA & $25 ; 36 ; 70$ \\
LDA & $74 ; 58 ; 63 ; 12$ \\
Correlation attribute feature selection & $88 ; 75 ; 20 ; 37 ; 65$ \\
Principal Component analysis & $52 ; 36 ; 42 ; 6 ; 67$ \\
Relief & $25 ; 38 ; 68 ; 71$ \\
\hline
\end{tabular}

TABLE 3 Output classifiers.

\begin{tabular}{|c|c|c|c|c|c|}
\hline Model Name & Method & Accuracy & Sensitivity & Specificity & F-Score \\
\hline \multirow{5}{*}{$\begin{array}{l}\text { Recurrent Fuzzy } \\
\text { Neural Network }\end{array}$} & DEA & 63.7 & 66.76 & 64.57 & 0.6345 \\
\hline & LDA & 72.5 & 74.45 & 73.34 & 0.7244 \\
\hline & Correlation & 98.43 & 99.54 & 97.63 & 0.9828 \\
\hline & PCA & 67.1 & 69.23 & 68.17 & 0.6701 \\
\hline & Relief & 56.5 & 59.43 & 58.22 & 0.5643 \\
\hline
\end{tabular}

TABLE 4 Comparison with previous works.

\begin{tabular}{lc}
\hline Method & Accuracy \\
\hline Simple KNN & $87.00 \%$ \\
CNN & $80.60 \%$ \\
Fuzzy KNN & $94.19 \%$ \\
BP-NN & $98.00 \%$ \\
Random Forest & $84.44 \%$ \\
SVM with a linear kernel & $83.88 \%$ \\
GA based trained RFNN & $97.78 \%$ \\
ANN-Fuzzy-AHP & $91.10 \%$ \\
Correlation-based trained RFNN & $98.43 \%$ \\
\hline
\end{tabular}

Table 3 shows the results of these models by separating the feature selection algorithms. By comparing Table 3 it can be concluded that the recurrent fuzzy neural network has the best performance by choosing the correlation feature selection than other methods because it has better evaluation rates.

In this situation, correlation-based feature selection reaches the best results because this method ranks features according to a heuristic evaluation function based on correlations. The function evaluates subsets made of attribute vectors, correlated with the class label but independent of each other. The CFS method assumes that irrelevant features show a low correlation with the class and, therefore, should be ignored by the algorithm. On the other hand, excess features should be examined, as they are usually strongly correlated with other attributes. The criterion used to assess a subset of 1 features can be expressed as follows:

$$
M_{S}=\frac{l \bar{t}_{c f}}{\sqrt{l+l(l-1) \bar{t}_{f f}}}
$$

Where:

$M_{S}$ is the evaluation of a subset of $\mathrm{S}$ consisting of 1 features.

$\bar{t}_{c f}$ is the average correlation value between features and class labels.

$\bar{t}_{f f}$ is the average correlation value between two features.

It means that, in this research, features $88 ; 75 ; 20 ; 37 ; 65$, which CFS selects have the best impact on the operation of RFNN.

For more discussion on this evaluation, this research compares with other previous works in Table 4 The testing set performance of this research on the UCI dataset compared with simple KNN ${ }^{[7]}, \mathrm{CNN}^{[8}$, Fuzzy KNN and BP-NN in ${ }^{[6}$, Random Forest ${ }^{[13}$, SVM with a linear kernel ${ }^{[5]}$, GA-based trained RFNN approach ${ }^{[4]}$ and ANN-Fuzzy-AHP ${ }^{[3]}$. Based on this comparison, the correlation-based trained RFNN approach has the best accuracy than those methods.

It can be concluded from Table 4 that, although the RFNN network has high quality in solving many problems, in this case of research, merging it with correlation feature selection improves the system's accuracy. 


\section{5 | CONCLUSION}

This study used the approach of training RFNN by five kinds of feature selection methods on the UCI Cleveland dataset to diagnose heart disease. Feature selection methods used in this study are DEA, LDA, CFS, PCA, and Relief. The result of the approach was evaluated with accuracies of the training set, testing set, and overall. The results of this study with CFS feature selection achieved a testing set accuracy of 98.43For future study, using different data sets from other sources may also be useful for testing the proposed system performance and handling this study with medical experts to include different attributes that can affect the method's decision capabilities.

\section{CREDIT}

Shirin Kordnoori: Investigation, Software, Conceptualization, Writing - Original Draft. Hamidreza Mostafaei: Formal analysis, Supervision, Writing - Review and Editing. Mohsen Rostamy-Malkhalifeh: Data Curation, Writing - Review and Editing. Mohammadmohsen Ostadrahimi: Data Curation, Writing - Review and Editing. Saeed Seyed Agha Banihashemi: Validation, Writing - Review and Editing.

\section{References}

1. Rakhshan SA. Efficiency ranking of decision making units in data envelopment analysis by using TOPSIS-DEA method. Journal of the Operational Research Society 2017;68(8):906-918.

2. Barat M, Tohidi G, Sanei M, Razavyan S. Data envelopment analysis for decision making unit with nonhomogeneous internal structures: An application to the banking industry. Journal of the Operational Research Society 2019;70(5):760769.

3. Samuel OW, Asogbon GM, Sangaiah AK, Fang P, Li G. An integrated decision support system based on ANN and FuzzyAHP for heart failure risk prediction. Expert Systems with Applications 2017;68:163-172.

4. Uyar K, Ilhan A. Diagnosis of heart disease using genetic algorithm based trained recurrent fuzzy neural networks. In: Procedia Computer Science, vol. 120; 2017. p. 588-593.

5. Louridi N, Amar M, Ouahidi BE. Identification of Cardiovascular Diseases Using Machine Learning. In: 7th Mediterranean Congress of Telecommunications 2019, CMT 2019; 2019. p. 1-6.

6. Yadav SS, Jadhav SM, Nagrale S, Patil N. Application of Machine Learning for the Detection of Heart Disease. In: 2nd International Conference on Innovative Mechanisms for Industry Applications, ICIMIA 2020 - Conference Proceedings; 2020. p. 165-172.

7. Reddy PK, Reddy SK, Balakrishnan S, Basha SM, Poluru RK. Heart disease prediction using machine learning algorithm. International Journal of Innovative Technology and Exploring Engineering 2019;8(10):2603-2606.

8. Lin CH, Yang PK, Lin YC, Fu PK. On Machine Learning Models for Heart Disease Diagnosis. In: 2nd IEEE Eurasia Conference on Biomedical Engineering, Healthcare and Sustainability 2020, ECBIOS 2020; 2020. p. 158-161.

9. Sharma V, Rasool A, Hajela G. Prediction of Heart disease using DNN. In: Proceedings of the 2nd International Conference on Inventive Research in Computing Applications, ICIRCA 2020; 2020. p. 554-562.

10. Li JP, Haq AU, Din SU, Khan J, Khan A, Saboor A. Heart Disease Identification Method Using Machine Learning Classification in E-Healthcare. IEEE Access 2020;8:107562-107582.

11. Magesh G, Swarnalatha P. Optimal feature selection through a cluster-based DT learning (CDTL) in heart disease prediction. Evolutionary Intelligence 2021;14(2):583-593.

12. Revathi KR, Kavitha KK. Comparison of classification techniques on heart disease data set. International Journal of Advanced Research in Computer Science 2017 dec;8(9):276-280. http://ijarcs.info/index.php/Ijarcs/article/view/4870. 
13. Buettner R, Schunter M. Efficient machine learning based detection of heart disease. In: 2019 IEEE International Conference on E-Health Networking, Application and Services, HealthCom 2019; 2019. p. 1-6.

How to cite this article: Kordnoori S., Mostafaei H., Rostamy-Malkhalifeh M., Ostadrahimi M., Banihashemi S.A., (2021), Diagnosis of Heart Disease Using Feature Selection Methods Based On Recurrent Fuzzy Neural Networks, IPTEK The Journal of Technology and Science, 32(2):64-73. 